EPJ Web of Conferences 116, 04003 (2016)

DOI: $10.1051 /$ epjconf/201611604003

(C) Owned by the authors, published by EDP Sciences, 2016

\title{
IceCube results from point-like source searches using 6 years of through-going muon data
}

\author{
Stefan Coenders ${ }^{\mathrm{a}}$ for the IceCube Collaboration ${ }^{\mathrm{b}}$ \\ Technische Universität München, Boltzmannstr. 2, 85748 Garching, Germany
}

\begin{abstract}
The IceCube Neutrino Observatory located at the geographic South Pole was designed to study and discover high energy neutrinos coming from both galactic and extragalactic astrophysical sources. Track-like events induced by charged-current muon-neutrino interactions close to the IceCube detector give an angular resolution better than $1^{\circ}$ above $\mathrm{TeV}$ energies. We present here the results of searches for point-like astrophysical neutrino sources on the full sky using 6 years of detector livetime, of which three years use the complete IceCube detector. Within 2000 days of detector livetime, IceCube is sensitive to a steady flux substantially below $E^{2} \partial \phi / \partial E=10^{-12} \mathrm{TeVcm}^{-2} \mathrm{~s}^{-1}$ in the northern sky for neutrino energies above $10 \mathrm{TeV}$.
\end{abstract}

\section{Introduction}

IceCube is a cubic-kilometer neutrino detector installed in the ice at the geographic South Pole [1] between depths of $1450 \mathrm{~m}$ and $2450 \mathrm{~m}$. Detector construction started in 2005 and finished in 2010. Neutrino reconstruction relies on the optical detection of Cherenkov radiation emitted by secondary particles produced in neutrino interactions in the surrounding ice or the nearby bedrock. During construction, partial detector configurations were operational taking data with 40 strings from April 2008, 59 strings from May 2009, and 79 strings from May 2010 on before IceCube was fully operational in May 2011 using 86 strings. Partial detector configurations are denoted by $I C$, followed by the number of active strings, e.g., IC59 for the 59 string season.

The observation of high-energy astrophysical neutrinos by IceCube using events starting in the detector volume [2], and with up-going muons mainly created in charged-current muon-neutrino interactions outside the detector [3], adds impetus to the searches for astrophysical neutrino sources. The first detection of a cosmic neutrino point-like source will represent a major breakthrough and will provide a smoking gun for cosmic ray acceleration. In hadronic interactions, neutrinos are produced alongside photons and charged particles, but due to their neutral charge and low interaction crosssection, neutrinos are neither deflected by magnetic fields, nor absorbed by interstellar media. Here, we present the results of searches for time-independent neutrino emission using both a scan of the fullsky and a dedicated search using known high-energy $\gamma$-ray objects.

\footnotetext{
ae-mail: coenders@icecube.wisc .edu

${ }^{b}$ http://icecube.wisc.edu
}

This is an Open Access article distributed under the terms of the Creative Commons Attribution License 4.0, which permits unrestricted use, distribution, and reproduction in any medium, provided the original work is properly cited. 

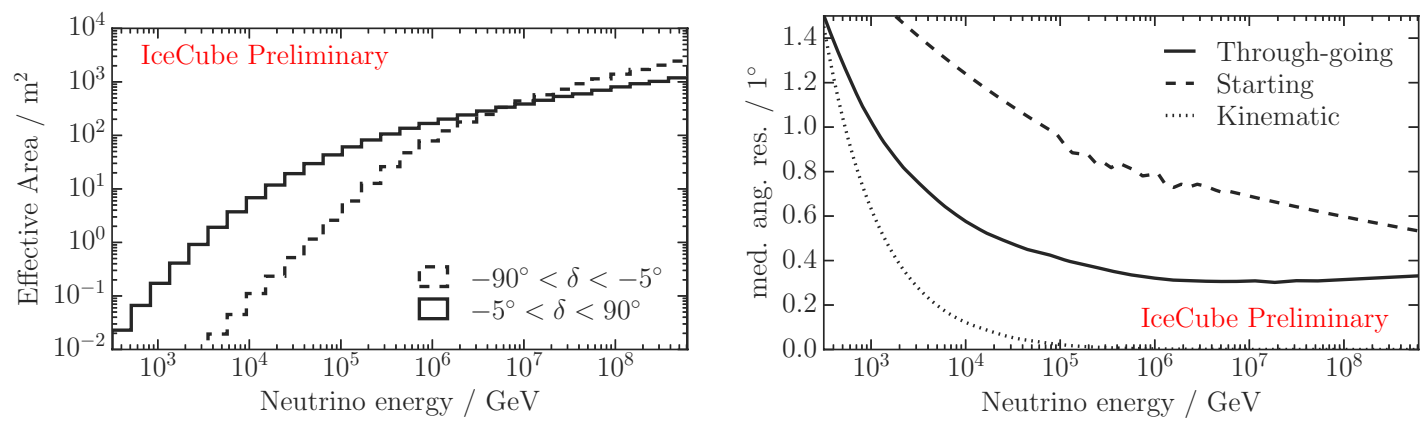

Figure 1. Effective area (left) and angular uncertainty (right) for $v_{\mu}+\bar{v}_{\mu}$ in this analysis. The effective area is shown for up-going (solid) and down-going (dashed) events separately. Angular resolutions are shown for tracks traversing (solid) and starting (dashed) in the detector. The intrinsic kinematic angle of muon and parent neutrino in $v_{\mu}$ charged current interactions is shown as well (dotted).

\section{Point source searches in IceCube}

The analysis presented here is a search for neutrino point-like sources using a sample chiefly made up of muon events entering the detector, but also identifying starting muon tracks identified with veto techniques [2]. Muons are created by charged-current $v_{\mu}+\bar{v}_{\mu}$ interactions and travel several kilometers. Thus, IceCube collects large amounts of neutrino interactions far outside the detector, increasing the statistics largely compared to starting event searches.

Using a multivariate selection technique, approximately 70000 neutrinos are collected in the northern sky each year, and a similar number of muons in the southern sky. The event sample in the northern sky consists of atmospheric neutrinos that form an irreducible background. In the southern sky, the largest background are bundles of atmospheric muons. The atmospheric spectrum is softer than the one expected for astrophysical neutrinos. Thus, the background rate is reduced by requiring only the highest-energy tracks to pass the event selection, yielding an event sample that is efficient for neutrino sources from above $\mathrm{PeV}$ up to $\mathrm{EeV}$ energies. More details about the event selection can be found at [4-6]. The graphs in Fig. 1 show the effective area (left) and median angular resolution (right) of $v_{\mu}+\bar{v}_{\mu}$ events detected by IceCube versus neutrino energy after the event selection. Above TeV energies, the muon direction is well correlated with the parent neutrino interaction. Overall, a neutrino reconstruction accuracy better than $0.8^{\circ}$ is achieved above $\mathrm{TeV}$ energies.

Starting events using veto techniques as in [2] provide a complementary sample compared to through-going muons in the southern sky. Using an active veto, the atmospheric background of incoming muons is significantly removed, which allows energy regions as low as $\sim 100 \mathrm{TeV}$ to be probed while retaining an angular resolution below $1^{\circ}$. These energy regions are not accessible using through-going muons in the southern sky, due to the large background.

The event sample is tested for clustering of events by maximizing the unbinned likelihood

$$
\mathcal{L}=\prod_{i} \prod_{j \in i}\left(\frac{n_{s}^{j}}{N} S\left(\left|\mathbf{x}_{j}-\mathbf{x}_{S}\right|, E_{j}, \sigma_{j} ; \gamma\right)+\left(1-\frac{n_{s}^{j}}{N}\right) B\left(\delta_{j}, E_{j}\right)\right)
$$

for all events $j$ (total sum $N$ ) using partial and full detector configurations $(j \in$ $\{$ IC40, IC59, IC79, IC86\}), the probability of an event to be signal $\mathcal{S}$ or background $\mathcal{B}$ is used to optimize for the number of associated source events $n_{s}^{j}$ in that sample and the source spectral index $\gamma$. Signal events at position $\mathbf{x}_{j}$ are assumed to cluster at the source position $\mathbf{x}_{S}$ given their reconstruction uncertainty $\sigma_{j}$. The background probability is estimated using an off-source region of events at the same declination $\delta_{j}$. In addition to spatial clustering, the energy is used to distinguish signal 


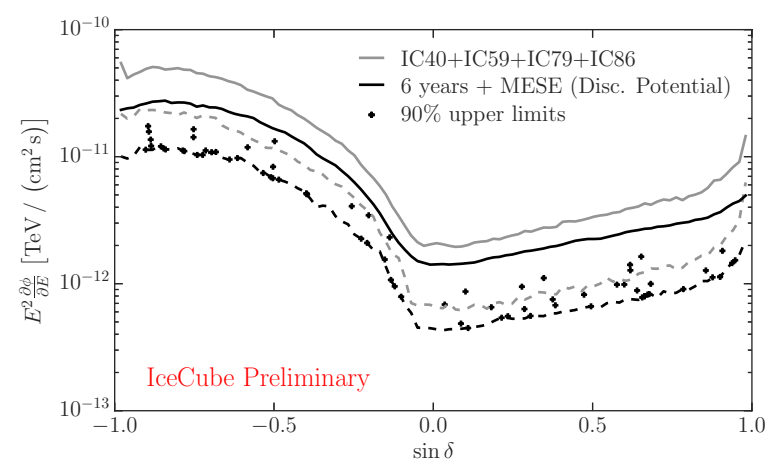

Figure 2. IceCube sensitivity (dashed) and discovery potential (solid) for point-like $v_{\mu}+\bar{v}_{\mu}$ sources using six years of detector exposure. In addition, medium energy starting events (MESE) are used in the southern sky. Black crosses indicate $90 \%$ upper limits for selected source candidates assuming an unbroken $E^{-2}$ power-law. Grey lines show the same properties of IceCube's previous point source search using four years of exposure [5].

from background. Astrophysical sources are expected to produce high-energy neutrinos, whereas the atmospheric background has a soft energy spectrum. For each spot on the sky, the test statistic $\log \Lambda=-2 \log \frac{\mathcal{L}\left(n_{s}, \gamma\right)}{\mathcal{L}\left(n_{s}=0\right)}$ with respect to the null hypothesis of no clustering $\left(n_{s}=0\right)$ is calculated.

The sensitivity and discovery potential of the six year event sample is shown in Fig. 2. Compared to the previous analysis [5], a gain is observed for all points in the sky. The effect of including starting events can be seen particularly in the extent that the bound is lowered in the southern hemisphere meaning that IceCube reaches the sensitivity of ANTARES [7]. However, the accessible energy range in the southern sky is different for both experiments, where IceCube covers the region above $100 \mathrm{TeV}$, while ANTARES is much more sensitive at lower energies, for example cut-off $E^{-2}$-spectra [8]. Dedicated searches using solely events starting in IceCube give additional sensitivity in that regime [9].

Using the test statistic of the unbinned likelihood (1), the entire sky is probed for significant clustering above background expectation. For this all-sky scan, in both northern and southern sky the point with most clustering is reported. To account for the look elsewhere effect, this procedure is redone for pure background data-sets, where the right ascension of all events is scrambled uniformly. Hence, the pre-trial $\mathrm{p}$-value of these points is trial-corrected to obtain the true significance.

In order to reduce the large effect of the trial-factor from the unbiased all-sky scan, a source list of prominent $\gamma$-ray sources was selected $a$-priori to be analyzed individually. This source list consists of sources of different types, for example pulsar wind nebulae in the galactic plane, and extra-galactic active galactic nuclei like BL Lac.

\section{Results}

In the analysis of over 600000 events distributed over the full sky within 6 years of IceCube livetime, no significant clustering was observed. Every cluster seen is compatible with expectations from pure background fluctuations.

In the northern sky, the most significant clustering (hot spot) is located at $\alpha=249.6^{\circ}, \delta=63.6^{\circ}$. The likelihood is fit to $n_{s}=29$ and $\gamma=2.1$ corresponding to a pre-trial $\mathrm{p}$-value of $-\log _{10} \mathrm{p}=5.75$. After accounting for the trial factor, the p-value is corrected to $35 \%$. In the southern sky, the hot spot is located at $\alpha=300.4^{\circ}, \delta=-33.2^{\circ}$ and yields a post-trial p-value of $87 \%$ at $n_{s}=19, \gamma=2.3$.

The analysis of the promising sources found that the source PKS1406-076 (a flat spectrum radio quasar) is associated with the highest neutrino clustering $\left(n_{s}=12.9\right)$. The observation is compatible with background (p-value 5.1\%) and upper limits are set for all sources. The limits are indicated 
with crosses at the source declination in Fig. 2. The 90\% upper limit for PKS1406-076 yields $2.32 \times 10^{-12} \mathrm{TeV} / \mathrm{cm}^{2} \mathrm{~s}$.

From the observed $\gamma$-ray spectra of sources, models of the expected neutrino spectra can be built. Two models from [10] and [11] are tested for the Crab Nebula. The model from [10] uses the connection between $\gamma$-rays and neutrinos produced in pion decays while the model in [11] is derived from Monte Carlo simulation of inelastic p-p scattering in the source. For the model of [11], the IceCube upper limit is two thirds of the model prediction, whereas the limit for the model from [10] is still 30\% above the prediction.

Note, that the sensitivity of IceCube with respect to that model matches the prediction in this analysis, but an observed fluctuation $\left(n_{s}=6.8\right)$ at the position of the Crab Nebula gives a higher upper limit. Within the next years, IceCube will be able to tighten the bounds on that model as well and thus probe the amount of hadronic acceleration which is occurring in addition to purely leptonic processes.

\section{Conclusion}

The analysis presented here, based on 6 years exposure and more than 600000 events in a throughgoing track sample, did not find any clustering above the background expectation. The previous search [5] was extended by an additional two years of livetime in the full detector configuration, significantly improving the discovery potential of the analysis. For the first time, the sensitivity to steady neutrino sources significantly surpasses $10^{-12} \mathrm{TeV} / \mathrm{cm}^{2}$ s over most of the northern sky.

IceCube puts stringent upper limits on the neutrino flux coming from various sources in the northern sky, constraining models of hadronic acceleration due to the non-detection of neutrinos from the sources. For some of the sources, IceCube has capabilities to test models and probe the amount of hadronic versus leptonic acceleration.

\section{References}

[1] A. Achterberg et al. (IceCube), Astropart.Phys. 26, 155 (2006)

[2] M.G. Aartsen et al. (IceCube), Phys.Rev.Lett. 113, 101101 (2014)

[3] M.G. Aartsen et al. (IceCube), Phys. Rev. Lett. 115, 081102 (2015)

[4] M.G. Aartsen et al. (IceCube), Astrophys.J. 779, 132 (2013)

[5] M.G. Aartsen et al. (IceCube), The Astrophysical Journal 796, 109 (2014)

[6] S. Coenders (IceCube), PoS(ICRC2015)1047 (2015)

[7] S. Adrian-Martinez et al. (ANTARES), Astrophys. J. 786, L5 (2014)

[8] S. Adrian-Martinez et al. (IceCube, ANTARES) (2015), 1511.02149

[9] S. Euler (IceCube), IceCube results from low-energy point-source searches in the southern hemisphere, these proceedings (2015)

[10] A. Kappes et al., The Astrophysical Journal 656, 870 (2007)

[11] E. Amato, D. Guetta, P. Blasi, Astron.Astrophys. 402, 827 (2003) 